\title{
Postinfectious Autoimmune Syndrome as a Key Factor in Chronization of the Infectious Disease
}

\author{
Natalia Cherepahina, Murat Agirov, Jamilyia Tabaksoeva, \\ Kusum Ahmedilova and Sergey Suchkov \\ First Moscow State Medical University \\ Russian State Medical University \\ Russia
}

\section{Introduction}

Disturbances in immune tolerance provoke autoimmune aggression, i.e., a specific immune response to auto-Ags with subsequent development of an autoimmune syndrome or an autoimmune disease (Suchkov et al., 2007).

A crucial role in formation of autoimmune syndromes and progression of autoimmune diseases is played by inborn (in the first place, HLA-associated) predisposition coupled with impaired immune responsiveness of the invaded organism. Noteworthy, initiation and progression of autoaggressive reactions cannot be triggered without preliminary activation of signaling reaction cascades, which include:

i. polyclonal activation of autoreactive cytotoxic $\mathrm{T}$ lymphocytes (CTL) by super-Ag (multimolecular protein complexes composed of microbial Ags, Ags and/or haptens of the carrier or intermediary drug-related metabolites) demonstrating broad spectrum of epitopes;

ii. release of sequestered or intramolecular (cryptic) autoepitopes after the tissue damage or organ injures during the inflammatory process;

iii. anti-idiotypic Ab formation that can damage own tissue and promote autoagression;

iv. effect of mimicking epitopes (microbial Ags cross-reacting with autoepitopes of human tissues and organs).

Of particular interest in this respect is so-called molecular mimicry. Its biological mechanism is based on cross-reactivity, i.e., ability of the infected organism to cross-react, by virtue of structural homology between its auto-Ags and microbial Ags, with the microbial antigen thereby triggering miscellaneous immune reactions. Under these conditions, the role of autoaggressors is played simultaneously by two different groups of Ags, namely, mimicking Ags of the microbial pathogen and patient's own autoAgs. Their interactions form the clinical picture of the postinfectious autoimmune syndrome (PIFA), one of main clinical variants of syndromeal immune pathology (Paltsev et al., 2009a).

Today, the key role of the immune system in the pathogenesis of chronically relapsing infectious diseases (CRID) leaves no doubt. Their clinical course is controlled by an immense variety of factors and their combinations among which the immunologic syndrome (IS) reflecting the origin and severity of disturbances in immune homeostasis occupies a special niche. The concept of IS is not new in principle and is widely met in the current literature. 
However, the term "clinico-immunological syndrome" (CIS) is far less explicit and needs to be supplemented with a pathogenetically rationalized, clinically significant formulaic definition encompassing the tremendous body of evidence accumulated thus far in the modern literature (Paltsev et al., 2009b) (Fig. 1).

- Causal factor

\section{CLINICAL CRITERIA}

- Lingering or chronic course of inflammatory processes (irrespective of localization) associated with frequent relapses

- Activation of conditionally pathogenic microflora, mixed infections; changes in the infectious pathogen during progression of the disease; involvement of other internal organs in autoimmune process

- Resistance to antibacterial therapy

\section{CRITERIA OF STRUCTURAL IMMUNODEFICIENCY}

- Clinical picture

- Deterioration of parameters reflecting populational magnitude and functional activity of lymphocytes, their subpopulations and non-specific protective factors to levels below the physiological level

- Diagnostically significant deterioration of 2-3 parameters in one component of the immune system or associated disturbances

Fig. 1. Clinical and immunological criteria of PIFSI

PIFSI - postinfectious secondary immunodeficiency syndrome.

In this section, we shall consider one of the most important clinical aspects of CIS, viz., postinfectious CIS (PICIS) whose role for practitioners in clinical medicine can hardly be overestimated. PICIS being a form of secondary (syndromal) immune pathology associated with the underlying (infectious) disease is provoked by a variety of factors including infectious pathogens of various etiology, clinical progression and complication of the disease proper, or inadequately applied antimicrobial therapy. The most common forms of this syndrome are as follows:

i. postinfectious secondary immunodeficiency syndrome (PIFSI);

ii. (ii) postinfectious autoimmune syndrome (PIFA);

iii. autoimmune syndrome coupled with postinfectious secondary immunodeficiency (PIFASID) (Suchkov et al., 2004).

Predisposition to one or another form of syndromeal immune pathology depends on a great number of genetically determined factors, which play a key role in the formation of patient's own immune resources. Its functional activity is controlled by coordinated functioning of innate and adaptive immune mechanisms; however, their role in the development and chronization of infectious processes is still open to question, which strongly impedes the construction of state-of-art immunopathogenetic models (Fig. 2). 


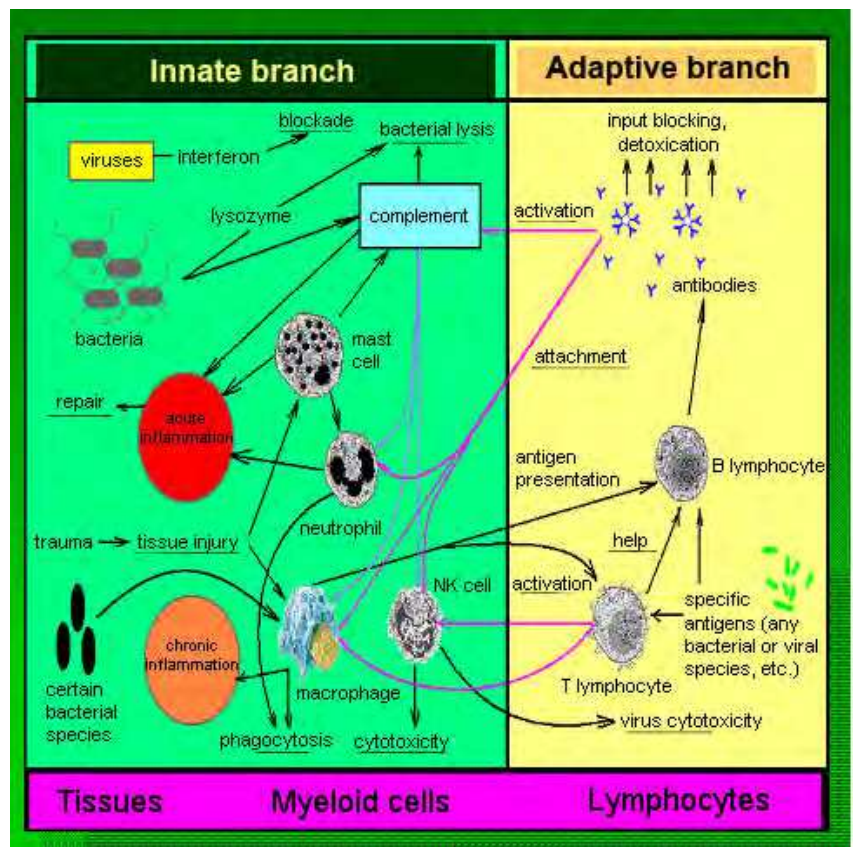

Fig. 2. The innate and adaptive branches of immunity.

In this context, analysis of major immunopathologic manifestations in patients with PICISrelated chronically relapsing infectious diseases and construction of basic algorithms for state-of-art immunogenetic diagnostic protocols becomes a prime target for clinical medicine.

Human immune system is a complex physiological mechanism whereby the human organism protects itself from exogenous etiopathogenic attacks. Its functional activity is provided by two types of protective immune mechanisms, one of which is specific and the other one is nonspecific. The main outcome of the immune response to etiopathogenic attacks is formation of two populations of regulatory $\mathrm{T}$ helper cells (Th cells). The Th population is further subdivided into Th1 cells responsible for activation of effector links of cell-mediated immunity (macrophages and cytotoxic T lymphocytes/CTL) and Th2 cells exerting control over antibody (AB) production (McGuirk \& Mills, 2002) (Fig. 3).

However, the key factor in determining a particular type of the immune response and, correspondingly, a particular form of CIS, is localization (extracellular or intracellular) of the etiopathogen (Fig. 4).

The latter circumstance is of particular importance from both pathogenetic and clinical points of view, since the majority of currently known pathogenic microorganisms can escape from immune control and, in doing so, change the scenario of genetically programmed immune responsiveness thereby provoking unpredictable complications for the patient and hindering physician's attempts to implement adequate treatment strategies (Azikury, 1985; Aitpaev \& Seisembekov, 1987).

Two major disturbances in immune responsiveness are presently recognized as causal factors in chronization of infectious diseases and formation of PICIS: 


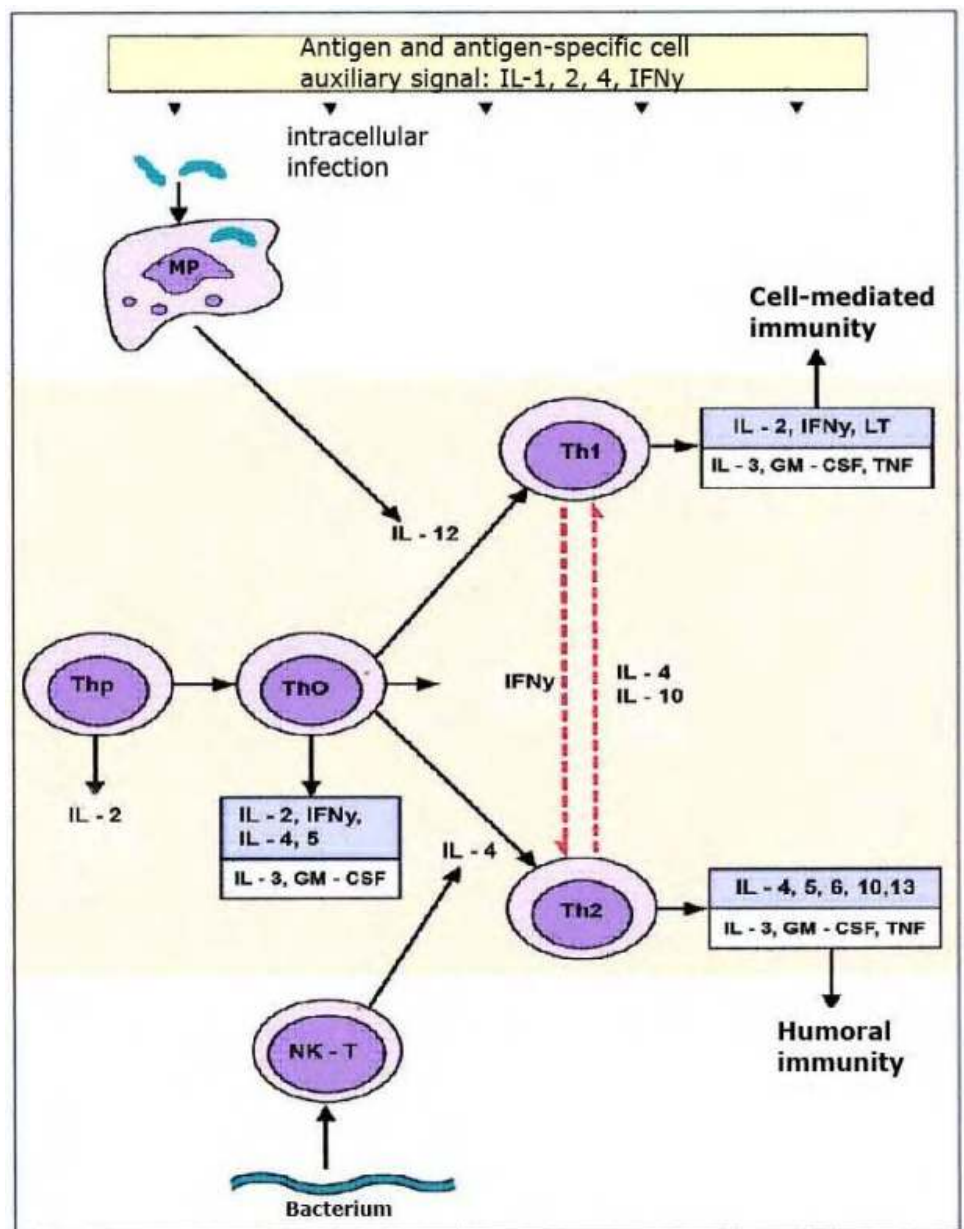

Note: In the presence of IL-4, precursor Th0 cells are transformed into Th2 cells whose main function consists in activation of humoral immunity and production of definite classes of cytokins, viz., IL-3, IL4, IL-5, IL-6, IL-10, IL-13, TNF, etc. Under the action of IL-12, Th0 precursors are transformed into Th2 cells stimulating the production of other cytokin populations, e.g., IL-2, IL-3, IFN $-\gamma$, TNF- $\alpha$, TNF- $\beta$, etc., able to activate cell-mediated immune responses. Other Th1/Th2 classes are represented by natural killer cells (NK cells), helper T cells (Th cells), granulocytic macrophageal colony-stimulating factors (GM-CSF), interferon (IFN), interleukin (IL), macrophages (MØ) and tumor necrosis factor (TNF).

Fig. 3. The pathways of formation of Th1/Th2 lymphocytes.

i. deficiency of effector links of immunity with predominant involvement of the T link (as in the case of isolated forms of PIFSI);

ii. disbalance of intercellular immunoregulatory mechanisms responsible for the formation of associated forms of syndromeal immune pathologies, e.g., PIFA and PIFASID). 


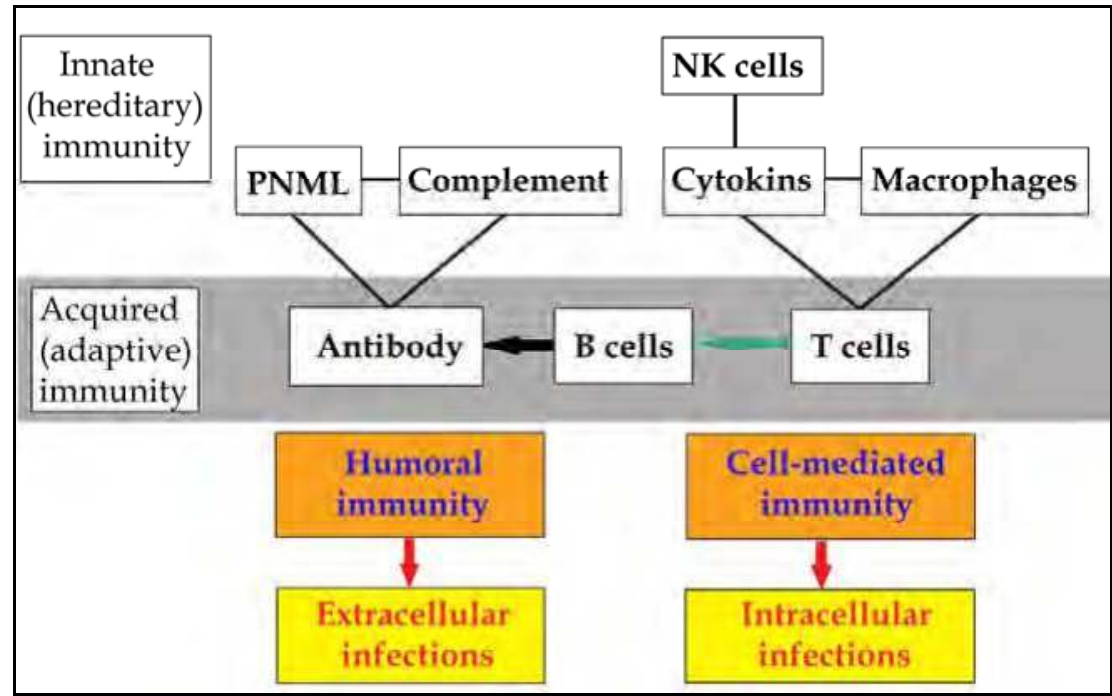

Note: PMNL and NK are polymorphonuclear leukocytes and natural killer cells, respectively.

Fig. 4 . The contribution of the innate and adaptive branches of immunity to control over intra- and extracellular infections.

\section{Clinical manifestations of PICIS in the framework of clinical models of CRID}

As targets for our investigation, we chose three classical models of CRID, namely, intracranial infectious inflammatory pathologies (ICIIP), chronic pyelonephrites (CPN) and myocardites (M). All these pathologies have one common feature (i.e., association with a concrete organ or a tissue), but differ from one another both topically and pathogenetically. Although the panel of immunologic disturbances varies substantially depending on the clinical form of PICIS, immune statuses of patients and clinical manifestations of the diseases are very similar (Antonov \& Tsinzerling, 2001; Borisov, 2000; Kukhtevich et al., 1997; Morozov, 2001; Paukov, 1996).

\subsection{Immunopathological factors as biomarkers and biopredictors of chronization of infectious diseases}

\subsubsection{Inflammation mediators as PICIS-related factors}

Emergence and accumulation, in patient's blood, of inflammation markers whose concentration reaches the highest level in patients with PIFA and degresses in the direction from PIFASID to PIFSI are the most common markers of chronization of infectious diseases and formation of syndromeal immune pathologies (Mazo et al., 2007; Litvinov et al., 2008; Zhmurov et al., 2000; Rumyantsev \& Goncharova, 2000).

\subsubsection{Abnormalities in the innate branch of immunity as a PICIS-related factor}

Miscellaneous shifts in the innate branch of immunity play a no less important role in chronization of infectious diseases. Thus, pronounced suppression of innate immune 
mechanisms is a salient feature of PIFSI, while PIFA and PIFASID are distinguished for disproportions in individual links of innate immunity and/or disbalance in the functional activity of its specific mechanisms (Bauer et al., 2001; Bingen-Bidois et al., 2002; Blackwell et al., 1987; Carballido et al., 2003; Dantzer \& Wollman, 2003).

Complement deficiency. In patients with PIFA and PIFASID, outbursts of activity in the C5 and C5a components of the complement are usually observed against the background of stable operation of the majority of other links of the immune system (PIFA) or pronounced disproportions between them (PIFASID).

Deficiency of phagocytosis and cytotoxicity mechanisms. To factors responsible for chronization of infectious diseases, one may relate oppositely directed changes in phagocytosis and cytotoxicity parameters. In PIFSI, both mechanisms are strongly suppressed, while in PIFA and PIFASID relative stability of certain components of both systems is concomitant with disproportions in other components.

Deficiency of dendritic cells. Dendritic cells (DCs) are among the most essential regulatory factors in the innate branch of immunity. In patients with PIFSI, the specific contribution of these cells is rather small, while in case of PIFASID and PIFA DCs play a prominent role and show a tendency for activation (Sanaev et al., 2008; Cherepakhina et al., 2009).

\subsubsection{Abnormalities in the adaptive branch of immunity as a PICIS-related factor}

Deficiency of $\mathrm{T}$ cell-mediated immunity. Among other disturbances in the adaptive branch of immunity, special attention should be given to differently directed changes in $\mathrm{T}$ cellmediated immunity. PIFSI, for example, is characterized by enhanced suppression of $\mathrm{T}$ cell functions resulting from disproportions in immunoregulatory components and massive apoptosis of $\mathrm{T}$ cells. In contrast, activation of T cell-mediated immunity is critical for PIFA and PIFASID, being more pronounced for the former and less pronounced for the latter.

Deficiency of humoral immunity. Suppression of humoral immunity is a characteristic feature of PIFSI, while PIFA and PIFASID are associated with its activation. The activating effect of quantitative and qualitative (functional) mechanisms of humoral immunity is especially apparent in PIFA, while in patients with PIFASID this effect is far less expressed.

Disproportions in the cytokin spectrum of the blood. PIFSI is associated with significant reduction of the population of antiinflammatory cytokins, while in PIFA this population is predominant. PIFASID is characterized by general disproportions in the cytokin spectrum at large (Cherepakhina et al., 2010a).

\section{PICIS and its main clinical forms}

\subsection{PIFSI}

The main clinical manifestations of PIFSI are related to disturbances in antimicrobial protective mechanisms due to deficiency of the innate branch of immunity and development of secondary immune pathologies in the adaptive branch of immunity. The latter manifest themselves as chronically relapsing infectious diseases of bacterial or mixed origin (Shogenov et al., 2006).

\subsection{PIFA}

During induction and progression of CRID, some autoreactive CTL cross-reacting with microbial antigens (Ags) in the paradigm of the infectious process undergo activation by 
hazardous factors including molecular mimicry (Khitrov et al., 2007a; Fujinami et al., 2006; Rose \& Mackay, 2000; Benoist \& Mathis, 2001). Its consequences are especially apparent during recognition of determinant autoAgs by $\mathrm{T}$ cells and subsequent formation of the PIFA syndrome (Fig. 5). The latter attack any target organ or tissue of the infected organism by a rocket mechanism. The risk of PIFA development increases dramatically with increasing incidence of infectious diseases and the panel of infecting pathogens (mixed infections).

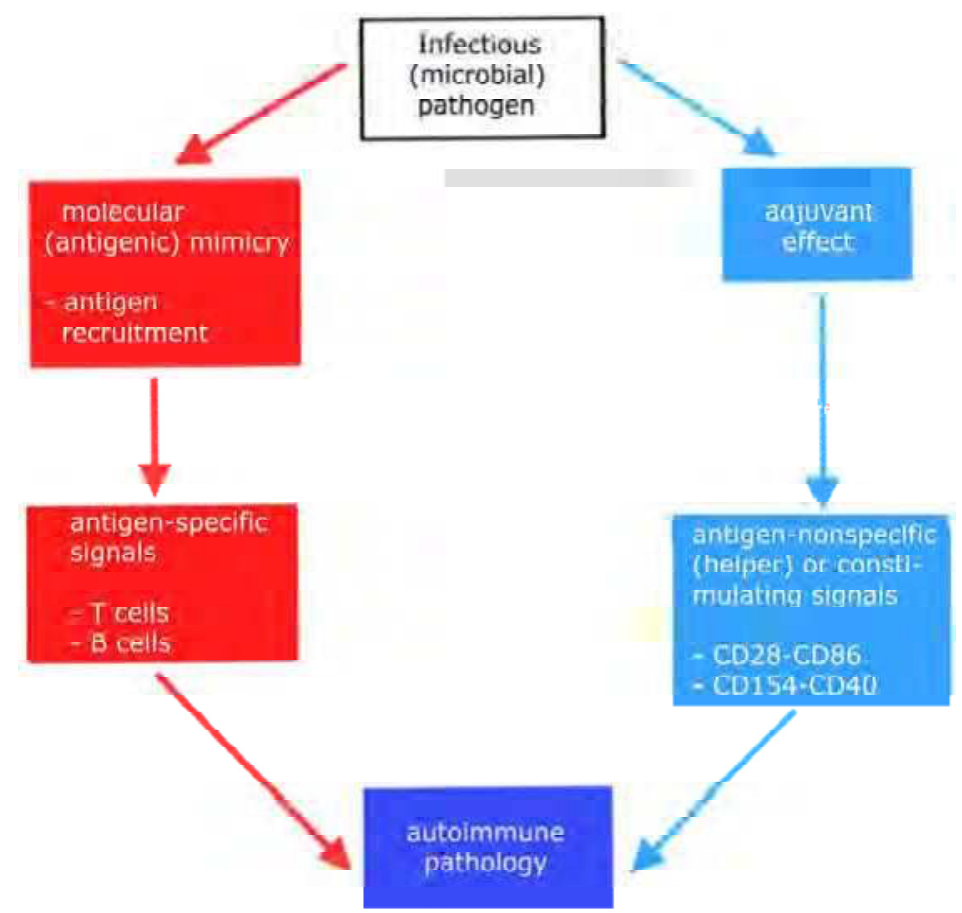

Note: The primary infectious (microbial) pathogen triggers a postinfectious autoimmune syndrome (PIFA) through activation of two different mechanisms: (i) depletion of intrinsic (antigenic) molecular mimicry pools of cross-reacting (mimicking) antigenic determinants of the infecting pathogen (red arrows); (b) generation, by the infectious pathogen, of antigen-nonspecific signals (blue arrows) able to induce inflammation and thus enhance immune responsiveness (so-called adjuvant effect).

Fig. 5. A schematic representation of the postinfectious autoimmune syndrome (PIFA).

There exist at least three different interpretations for the relatedness of the infectious process to the risk of PIFA in response to activation of autoreactive clones of $\mathrm{T}$ and B lymphocytes, namely: (i) stimulation by microbial superAgs; (ii) secretion of cryptic (intramolecular) autoAg determinants in response to cell damage induced by persisting infections and (iii) molecular mimicry. These pathogenetic mechanisms are not mutually exclusive and play a crucial role in definite (as a rule, early) steps of PIFA-related CRID. The main triggering factors in the PIFA initiation step are: (i) antigenic activity of the microbial pathogen and (ii) tropism of the microbial pathogen towards definite cell populations, organs and tissues as targets for its cytopathic effect (Vturin et al., 1994; Manges et al., 2004). 
Contrary to PIFSI, all classes of antimicrobial ABs (antibacterial, antiviral, antiparasitic, etc.) are morbid in PIFA. Although in the majority of patients the incidence and titers of antibacterial and antiviral ABs are more or less identical, in certain forms of CRID (e.g., CPN or $\mathrm{M}$ ) antiparasitic $\mathrm{ABs}$ are detected in highest titers, while in patients with other pathologies (e.g., ICIIP) they are absent. These findings can be attributed to clinical manifestations of the underlying diseases rather than to inadequate functioning of triggering mechanisms of PIFA) (Cherepakhina et al., 2010b).

Indeed, autoaggression provoked by insufficient coordination between two branches of immunity and hyperfunction of its adaptive branch is a dominant feature of PIFA. Its unique feature is a vast repertoire of antiorganic and antitissue autoABs responsible for multiseropositivity and specific autoimmune inflammation markers, e.g., anti-B7-HI autoABs) (Khitrov et al., 2007).

By illustration, antimyelin and antineuronal autoABs are usually associated with ICIIP. Patients with CPN contain predominantly anti-THG autoABs as highly specific markers of autoimmune inflammation in renal tissue, while the presence of anti-KM autoABs indicates AIM (Miller et al., 1970).

To the most informative models of PIFA one may relate autoimmune myocarditis (AIM), autoimmune encephalomyelitis (AEM), ICIIP, rheumatoid arthritis (PA), autoimmune hepatitis (AIHe), autoimmune colienteritis (AICE), autoimmune pancreatitis (AIPCT), autoimmune gastritis (AIGa), autoimmune (streptococcal) glomerulonephritis (AGN), CPN, etc.

Autoimmune myocarditis (AIM) usually develops in genetically predisposed individuals infected with the Coxsackievirus-3 virus (CVB3) and is one of the most typical manifestations of molecular mimicry. The presence, in circulating blood, of cardiomyosinautoreactive cytotoxic T lymphocytes (KM-autoreactive CTL) and anti-KM autoABs is prerequisite to AIM development. Their interactions in patients with PIFA or PIFASID initiate myocardial lesions in response to enhanced secretion of sequestered autoAgs (Shogenov et al., 2010) (Fig. 6).

In type I diabetes mellitus (DM I), insulitis develops in genetically predisposed individuals at the earliest (preclinical) stages of the disease (as a rule, against the background of infection with the Coxsackie-4 virus (CVB4)), and is further transformed into PIFA. This pathological process is mediated by autoreactive CTL and autoABs against islet autoAgs. Their coordinated functioning initiates the destruction (direct or indirect) of beta cells, e.g., through secretion of cytokins, generation of free radicals or apoptosis of beta cells, eventually resulting in PIFA or PIFASID.

The main causal factors in initiation of chronically relapsing autoimmune colienteritis (AICE) are mimicking AGs of microbial or dietary origin. These AGs are localized in the intestinal lumen where they activate immune cells of intestinal mucosa. Having penetrated into these cells, AGs begin to interact with tissue immunocytes (most frequently, with lymphocytes and DCs) thereby triggering adaptive immune responses. Innate immune resources also become activated under the stimulating effect of microbial products due to activation of specific surface receptors of intestinal epithelium. This reaction cascade stimulates the secretion of numerous cytokins and chemokins able to activate immunocytes of intestinal mucosa. Activation of antigen-presenting cells (APC) (e.g., DCs) initiate enhanced production of Th1 cells (Crohn's disease) or atypical Th2 cells (ulcerative colitis). In addition to major cytokins stimulating the activity of Th1 cells (IL-12, IL-18, etc.), activated macrophages give rise to a great diversity of antiinflammatory cytokins (IL-1, IL-6, 


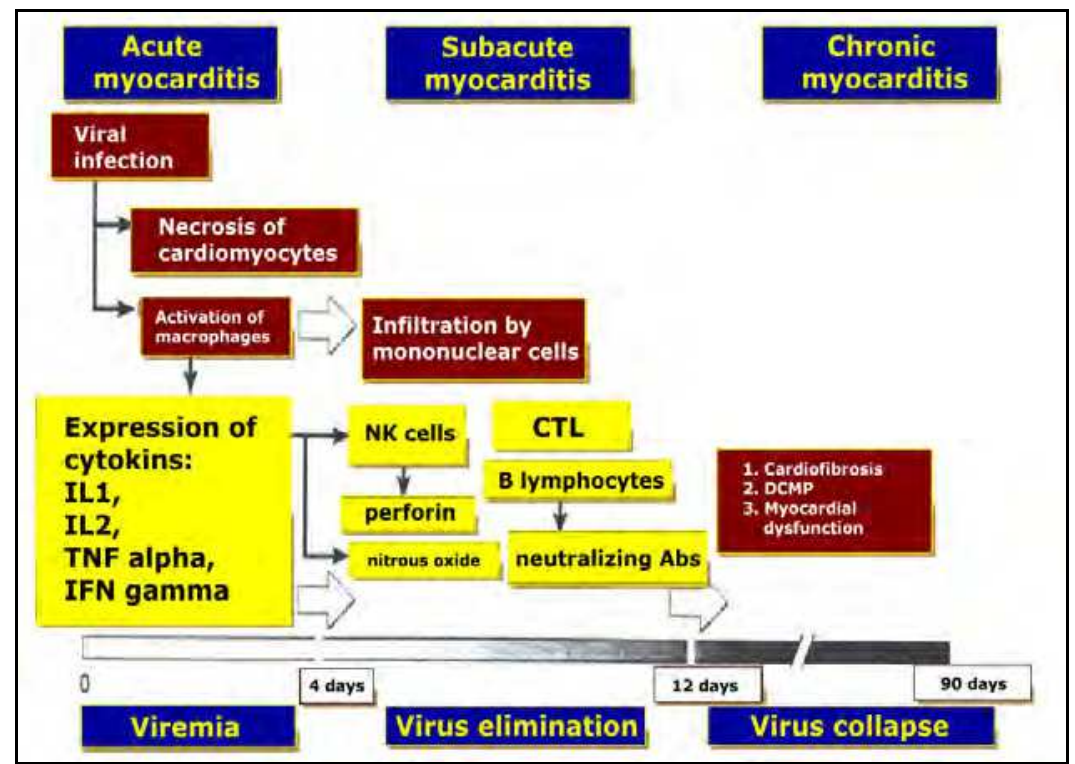

Note: AB - antibody; CTL - cytotoxic T lymphocyte; IFN - interferon; IL - interleukin; NK - natural killer cell; DCMП - dilated cardiomyopathy.

Fig. 6. Initiation and progression of myocarditis

TNF alpha, etc.) endowed with an ability to stimulate the activity of different cell populations (including endothelial cells) in inflammation foci by promoting enhanced migration of lymphocytes, fibroblasts and epithelial cells from the vascular network to inflammation niduses, which significantly deteriorates the clinical picture of autoimmune nidal inflammation (Khaitov \& Pinegin, 2000; Bach, 2005).

\subsection{PIFASID}

A salient feature of this syndrome is equal contribution of associated abnormalities to both branches of immunity. Its clinical picture is distinguished for mixed-type immunopathology, viz., autoimmune syndrome coupled with immunodeficiency and concurrent deterioration of antiinfectious protection.

\section{Associative correlation between clinical manifestations of PICIS and CRID}

The associativity between microbial infection and various immunopathological states with PICIS can be correlative or causal. In patients with CRID, syndromal forms of immune pathologies depend critically on the stage of the inflammatory process occurring in target organs or tissues and general chronization of the disease (Sanaev et al., 2007).

For example, early stages of CRID are concomitant with PIFSI (> 50\%), whereas the contribution of PIFA and PIFASID does not exceed 20\%. At the subsequent stages, the clinical picture is different, viz., the contribution of the autoimmune syndrome increases dramatically (to $50 \%$ at the intermediate stages (PIFA) and to $60 \%$ at the final stage (PIFASID). 
The correlation between the stage of CRID and the form of PICIS is also characterized by the involvement of an additional (third) component, viz., clinical form or variant of CRID. Here are several analytical examples related to:

1. clinical form of CRID. In patients with primary pyelonephritis (PPNP) and infectious myocarditis (IM), PIFSI is detected in $75 \%$ of cases, whereas in patients with secondary pyelonephrites (SPNP) and AIM the contribution of PIFSI is notably decreased (to 25\%) giving way to autoaggression (the contribution of PIFA and PIFASID increases to $60 \%$ and $85 \%$, respectively);

2. stage of CRID. At early stages ( $<3$ months for CPN and $<1$ month for myocarditis $(\mathrm{M})$ ), PIFSI is detected in $40 \%$ of cases; however, at later stages of CRID its share decreases appreciably, while that of autoimmune syndromes increases in contrast;

3. rate of progression and chronization of CRID. In patients with relapsing or rapidly progressing CRID (e.g., ICIIP or AIM), the contribution of PIFSI does not exceed 32$36 \%$, while the share of autoimmune syndromes reaches $80-100 \%$. In such patients, persistent forms of meningoencephalitis (e.g., ICIIP) or AIM associated with myocardial dystrophies are predominant.

These findings suggest that PIFSI is not only the outcome of the infectious process, but also represents a factor responsible for its lingering and chronically relapsing course. Further progression and chronization of CRID are controlled by postinfectious autoaggression factors, such as PIFA and PIFASID.

\section{Clinico-immunological criteria of PICIS and state-of-art immunogenetic diagnostic algorithms}

So far, there is no unique set of criteria for adequate assessment of immune statuses of patients with different forms of PICIS, most probably, due to immense diversity of clinical manifestations of syndromal immunopathologies and factors responsible for their emergence. Moreover, existing laboratory protocols for assessing immune statuses are nonspecific and do not include specific analyses of microbial pathogens (Vinnitskij, 2002; Kolesnikov et al., 2001; Cherepakhina et al., 2010c).

With this in mind and in order to procure adequate evaluation of many syndromal immune pathologies, we developed a series of clinical and immunologic tests and criteria for more precise diagnosis of PICIS. The criteria for constructing immunogram charts include:

i. screening of abnormalities in the innate branch of immunity (selective markers of phagocytosis, natural cytotoxicity (NCT), basic functional parameters of DC-and Agpresenting cells (APC) and complement components (if necessary);

ii. screening of abnormalities in the adaptive branch of immunity (selective markers of effector or regulatory links of the immune system, serotyping of blood elements for antiorganic and anti-tissue autoABs concurrently with identification of Abs against mimicking Ag determinants of infecting pathogens).

The main criteria in the etiotropic diagnosis step (design of microbial landscape maps) include:

i. identification and localization of microbial gene pools;

ii. serological profiles of antimicrobial ABs.

The novel diagnostic ideology is based on a combination of two categories of investigations:

i. pathogenetically oriented diagnosis of PICIS and (ii) etiotropic diagnosis of microbial pathogens as the main causal factors of PICIS. 
The most efficient technological strategies will be based on:

i. at the immunodiagnostics stage (cytofluorimetric analysis of processing and presentation of AGs on the surface of APC, monitoring of antiorganic and antitissue autoAB pools, analysis of metabolic profiles of individual cells, etc.);

ii. etiotropic diagnostics (combination of conventional techniques for culturing microbial cells with advanced molecular diagnostics strategies based on sequencing of microbial genomes, screening of biological fluids and tissues for antimicrobial ABs, etc.).

\section{Conclusion}

Morbidity from infectious pathologies (e.g., CRID), in the first place, those provoked by viruses, conditionally pathogenic ("opportunistic") microflora and/or pathogens endowed with atypical properties including muiltiple resistance to antibacterial drugs, is steadily increasing. Among other things, CRID-affected individuals are characterized by lowering general immune responsiveness concurrent with unusual forms of immune responses to the clinical course of the infectious pathology. Studies in this field including our own investigations established that PICIS is one of the most important clinical manifestations of CRID, since it determines, in many features, the progression and chronization of underlying pathologies and their possible complications. The monosyndromal dominant form of PICIS in patients with CRID is PIFSI. However, more than $30 \%$ of CRID patients suffer from more specific forms of PICIS concomitant with autoimmune aggression (PIFA) or from combined immunopathological forms (e.g., PIFASID).

Clinical forms of PICIS and, correspondingly, immunologic disturbances in patients with CRID correlate associatively with the clinical picture of the disease. It is not excluded that chronization of infectious inflammatory processes involves a general sequence of pathogenetically important factors, which differ in inner architechtonics of each of PICIS variants and thus demonstrate their high criterial significance.

Future progress in clinical immunology and immune biotechnology may open up fresh opportunities for introduction into routine clinical practice of advanced protocols for immunogenetic diagnostics of PICIS-related infectious diseases and design of state-of-art treatment-and-rehabilitation protocols based on the use of the most advanced immunogenetic tools and strategies.

\section{References}

Suchkov S.V.; Shogenov Z.S. \& Khitrov A.N. (2007). Postinfectious autoimmune syndrome: features of pathogenesis and modern protocols of clinical immunogenodiagnostics. Therapeutic Archives. Vol.79, No.4, (April 2007), pp. 71-76, ISSN 0040-3660

Paltsev M.A.; Cherepakhina N.E. \& Suchkov S.V. (2009). Postinfectious clinical immunologic syndrome: foundations of etiopathogenesis and strategy of immunogenodiagnostics. Bulletin of the Russian Academy of Medical Sciences. Vol.10, (October, 2009), pp. 25-31, ISSN 0869-6047

Paltsev M.A. Clinical and immune-mediated syndrome (CAIMS) in clinical practice: features and strategies in immune and molecular diagnostics. (2009). New Horizons in Allergy, Asthma \& Immunology, pp. 177-181, ISBN 978-88-7587-505-3, Dubai, UAE, April 24-27, 2009 
Suchkov S.V.; Blagoveschenskij S.V. \& Vinnitskij L.I. (2004). Modern aspects of immunopathogenesis and immunocorrection in patients with intracranial infectious inflammatory diseases. Allergology and Immunology. Vol.5, No.2, (2004), pp. 323-330, ISSN 1562-3637

McGuirk P. \& Mills K.H. Pathogen-specific regulatory T cells provoke a shift in the Th1/Th2 paradigm in immunity to infectious diseases. (2002). Trends in Immunology. Vol.23, No.9, (September 2002), pp. 450-455, ISSN 1471-4906

Azikury O.I. Cellular and humoral immunity at pyelonephritis. (1985). Urology and Nephrology. Vol.2, (March 1985), pp. 10-11, ISSN 0042-1154

Aitpaev B. K. \& Seisembekov T.Z. (1987). Cellular and humoral factors of nonspecific resistance and immunologic reactivity at chronic pyelonephritis. Therapeutic Archives. Vol.8, (August 1987), pp. 59-63, ISSN 0040-3660

Antonov V.P. \& Tsinzerling V.A. Contemporary status of the problem of chronic and slow neuroinfections. (2001). Archieves of Pathology. Vol.63(1), (January 2001), pp. 47-51, ISSN 0004-1955

Borisov I.A. Pyelonephritis. (2000). In: Nephrology, Tareeva I.E. (Ed.), pp. 383-399, Medicine, ISBN 5-225-04195-7, Moscow, Russia

Kukhtevich A.V.; Gordovskaya N.B. \& Kozlovskaya N.L. Pyelonephritis. Russian Medical Journal. Vol.5, No.23, (December 1997), pp. 54-62, ISSN 0869-7760

Morozov A.V. Chronic infection of urinary tract (pathogenesis, diagnostic and treatment principles). Russian Medical Journal. Vol.9, No.23, (December 2001), pp. 1074-1077, ISSN 0869-7760

Paukov V.S. Immunology and morphology of chronic inflammation. Achieves of Pathology. Vol.58(1), (January 1996), pp. 28-33, ISSN 0869-7760

Mazo E.B.; Vinnitskij L.I. \& Litvinov V.A. (2007). Chronic pyelonephritis: features of immunopathogenesis and its' clinical and diagnostical values. Therapeutic Archives. Vol.79(1), (January 2007), pp. 85-89, ISSN 0040-3660

Litvinov V.A.; Cherepakhina N.E. \& Suchkov S.V. (2008). Chronic pyelonephritis: features of immunopathogenesis and principles of clinical immunogenetic diagnostics. Physician. Vol.1, (January 2008), pp. 12-17, ISSN 0236-3054

Zhmurov V.A.; Oskolkov V.A. \& Malishevskij M.V. (2000). Correlation between immunogenetic markers and metabolic processes at chronic pyelonephritis. Urology. Vol.3, (2000), pp. 9-13, ISSN 0042-1154

Rumyantsev A.S. \& Goncharova I.S. Etiology and pathogenesis of pyelonephritis. Nephrology. Vol.4, No.3, (2000), pp. 40-52, ISSN 1561-6274

Bauer J.; Rauschka H. \& Lassmann H. Inflammation in the nervous system: the human perspective. Glia, Vol.36, No.2, (November 2001), pp. 235-243, ISSN 1098-1136

Bingen-Bidois M.; Clermont O. \& Bonacorsi S. Phylogenetic analysis and prevalence of urosepsis strains of Escherichia coli bearing pathogenicity island-like domains. Infect Immun. Vol. 70, No.6, (June 2002), pp. 3216-3226, ISSN 1098-5522

Blackwell C.C.; May S.J. \& Brettle R.P. Secretor state and immunoglobulin levels among women with recurrent urinary tract infections. J Clin Lab Immunol. Vol.22, No.3, (1987), pp. 133-137, ISSN 0141-2760

Carballido J.A.; Alvarez-Mon M. \& Olivier C. Inflammatory pathology in urology. Standardization. Actas Urol Esp. Vol.27, No.3, (March 2003), pp. 173-179, ISSN 02104806 
Dantzer R. \& Wollman E.E. Relationships between the brain and the immune system. J Soc Biol. Vol.197, No.2, (2003), pp. 81-88, ISSN 0037-766X

Sanaev A.O.; Kachkov I.A. \& Vinnitskij L.I. (2008). Immunomonitoring and immunorehabilitation in case of intracranial infectious inflammatory diseases. Russian Journal of Immunology. Vol.2(11), No.1, (2008), pp. 78-82, ISSN 1028-7221

Cherepakhina N.E.; Shogenov Z.S. \& Elbeik T. (2009). Postinfectious clinical-andimmunologic syndrome and its place in clinical practice. Therapeutic Archives. Vol.81(1), No.12, (December 2009), pp. 71-78, ISSN 0040-3660

Cherepakhina N.E.; Tabaksoeva D.A. \& Suchkov S.V. (2010). Associative relation of microbial factor and postinfectious clinical-and-immunologic syndrome in case of chronic relapsing diseases. Clinical Microbiology and Antimicrobial Chemotherapy. Vol.12, No.2, suppl.1, (2010), p. 54, ISSN 1684-4386

Shogenov Z.S.; Cherepakhina N.E. \& Suchkov S.V. (2006). Immunogenetic diagnostics and postinfectious immunodeficiency syndrome in physicians' practice. Clinical Laboratory Diagnostics. Vol.11, (November 2006), pp. 36-43, ISSN 0869-2084

Khitrov A.N.; Shogenov Z.S. \& Suchkov S.V. (2007). Molecular mimicry phenomenon and its place in postinfectious autoimmune syndrome (PIFA) pathogenesis. Molecular Medicine. Vol.4, (2007), pp. 24-32, ISSN 1728-2918

Fujinami R.S.; von Herrath M.G. \& Christen U. Molecular mimicry, bystander activation, or viral persistence: infections and autoimmune disease. Clin. Microbiol. Rev. Vol.19, No.1, (January 2006), pp. 80-94, ISSN 0893-8512

Rose N.R. \& Mackay I.R. Molecular mimicry: a critical look at exemplary instances in human diseases . Cell Mol. Life Sci. Vol.57, No.4 (April 2000), pp. 542-551, ISSN 1420-682X

Benoist C. \& Mathis D. Autoimmunity provoked by infection: how good is the case for T cell epitope mimicry? Nat. Immunol. Vol.2, No.9, (September 2001), pp. 797-801, ISSN 1529-2908

Vturin B.V.; Delektorskij V.V. \& Kovalchuk V.K. Pathogenic mechanisms of bacteria at different infections. (1994). Archives of pathology. Vol.56, No.5, (September 1994), pp. 10-15, ISSN 0004-1955

Manges A.R.; Dietrich P.S. \& Riley L.W. Multidrug-resistant Escherichia coli clonal groups causing community-acquired pyelonephritis. Clin Infect Dis. Vol.38, No.3, (February 2004), pp. 329-334, ISSN 0934-9723

Cherepakhina N.E.; Maksimenko D.M. \& Suchkov S.V. (2010). Microbial landscape and its value for the modern model of immunopathogenesis of chronic relapsing infectious diseases. Proceedings of the III World asthma \& COPD forum and World forum of pediatrics, pp. 99-102, ISBN 978-88-7587-558-9, Dubai, UAE, April 24-27, 2010

Khitrov A.N.; Shogenov Z.S. \& Tretyak E.B. (2007). Postinfectious immunodeficiency and autoimmunity: pathogenic and clinical values and implications. Expert Review of Clinical Immunology. Vol.3, No.3, (May 2007), pp. 323-331, ISSN 1744-666X

Miller T.E.; Smith J.W. \& Lehmann J.W. Autoimmunity in chronic experimental pyelonephritis. J Infect Dis. Vol.122, No.3, (September 1970), pp. 191-195, ISSN 13446304

Shogenov Z.S.; Akhmedilova K.A. \& Tabaksoeva D.A. Features of immunopathogenesis and chronization of myocarditis as the basis of immunogenetic diagnostic and 
immunogenetic monitoring protocols development. Russian cardiology journal. Vol.6(86), (November 2010), pp. 76-87, ISSN 1560-4071

Khaitov R.M. \& Pinegin B.V. Contemporary conceptions of safeguard of host organism against infection. Immunology. Vol.1, (January 2000), pp. 61-64, ISSN 0206-4952

Bach J.F. Infections and autoimmunity. Rev. Med. Interne. Vol.1, (October 2005), pp. 32-34, ISSN 0248-8663

Sanaev A.O.; Kachkov I.A. \& Vinnitskij L.I. (2007). Modern aspects of immunotherapy at intracranial infectious inflammatory diseases therapy by example of brain abscesses. Allergology and Immunology. Vol.8, No.4, (2007), pp. 384-387, ISSN 15623637

Vinnitskij L.I. Diagnostic facilities of contemporary immune technologies in surgical clinic. (2002) Allergology and Immunology. Vol.3, No.1, (2002), pp. 198-203, ISSN 1562-3637

Kolesnikov A.P.; Khabarov A.S. \& Kozlov V.A. (2001). Diagnostics and differentiated treatment of secondary immunodeficiencies. Therapeutic Archives. Vol.73(4), (April 2001), pp. 55-59, ISSN 0040-3660

Cherepakhina N.E.; Maksimenko D.M. \& Suchkov S.V. (2010). Strategy of immunotherapy and immunorehabilitation of chronic relapsing infectious. International Journal on Immunorehabilitation. Vol.12, No.2, (May 2010), p. 139, ISSN 1562-3629 


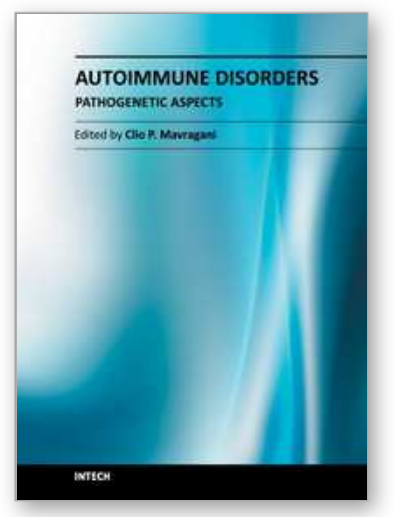

\author{
Autoimmune Disorders - Pathogenetic Aspects \\ Edited by Dr. Clio Mavragani
}

ISBN 978-953-307-643-0

Hard cover, 508 pages

Publisher InTech

Published online 26, October, 2011

Published in print edition October, 2011

The present edition entitled "Autoimmune disorders - Pathogenetic aspects" aims to present the current available evidence of etiopathogenetic insights of both systemic and organ specific autoimmune disorders, the crossover interactions among autoimmunity, cardiovascular morbidity and malignancy as well as novel findings in the exciting fields of osteoimmunology and immunology of pregnancy.

\title{
How to reference
}

In order to correctly reference this scholarly work, feel free to copy and paste the following:

Natalia Cherepahina, Murat Agirov, Jamilyia Tabaksoeva, Kusum Ahmedilova and Sergey Suchkov (2011). Postinfectious Autoimmune Syndrome as a Key Factor in Chronization of the Infectious Disease, Autoimmune Disorders - Pathogenetic Aspects, Dr. Clio Mavragani (Ed.), ISBN: 978-953-307-643-0, InTech, Available from: http://www.intechopen.com/books/autoimmune-disorders-pathogenetic-aspects/postinfectious-autoimmunesyndrome-as-a-key-factor-in-chronization-of-the-infectious-disease

\section{INTECH}

open science | open minds

\section{InTech Europe}

University Campus STeP Ri

Slavka Krautzeka 83/A

51000 Rijeka, Croatia

Phone: +385 (51) 770447

Fax: +385 (51) 686166

www.intechopen.com

\section{InTech China}

Unit 405, Office Block, Hotel Equatorial Shanghai

No.65, Yan An Road (West), Shanghai, 200040, China 中国上海市延安西路65号上海国际贵都大饭店办公楼 405 单元

Phone: +86-21-62489820

Fax: $+86-21-62489821$ 
(C) 2011 The Author(s). Licensee IntechOpen. This is an open access article distributed under the terms of the Creative Commons Attribution 3.0 License, which permits unrestricted use, distribution, and reproduction in any medium, provided the original work is properly cited. 